\title{
Inferior vena cava dimensions in patients with acute kidney injury
}

\author{
Andres Yepes-Hurtado MD, Sabry Omar MD, Khalid Monzer MD, \\ Raed Alalawi MD, Kenneth Nugent MD
}

\begin{abstract}
Background: Volume contraction frequently contributes to the development of acute kidney injury. The rapid assessment of volume status in patients with acute kidney injury could improve decision making and outcomes.

Methods: The maximum and minimum diameters and percent collapsibility of the inferior vena cava (IVC) were measured in 30 patients admitted to the medical intensive care unit with laboratory evidence of acute kidney injury. These measurements were made on the day of admission and 24 hours following admission. Information about age, gender, body mass index, serum creatinine levels, and fluid balances was recorded.

Results: This study included 30 patients with a mean age is $62.4 \pm 16.0$ years. The mean initial creatinine was $4.3 \pm 4.2 \mathrm{mg} / \mathrm{dL}$ (range: $1.7 \mathrm{mg} / \mathrm{dL}$ to $22.1 \mathrm{mg} / \mathrm{dL}$ ). The mean fractional excretion of sodium was $2.06 \pm 2.65 \%$. The mean maximum diameter of inferior vena cava was $1.8 \pm 0.5 \mathrm{~cm}$ with the range is $0.4-2.65 \mathrm{~cm}$. The mean percent collapse was $32 \pm 20 \%$. Five patients had evidence of hypovolemia using guidelines from the American Society of Echocardiology; 6 patients had evidence of hypervolemia. Nineteen patients had measurements between these 2 categories. There is no significant change in mean diameters following fluid administration for 24 hours. An initial IVC diameter of $0.94 \mathrm{~cm}$ predicted $\geq 30 \%$ collapsibility with an area under the curve is 0.748 .

Discussion: Patients with acute kidney injury based on laboratory measurements had evidence for hypovolemia, euvolemia, and hypervolemia based on IVC measurements. There was no consistent change in IVC dimensions following fluid administration, even though the creatinine fell in most patients. Simple bedside measurements of IVC dimensions can facilitate fluid administration decisions but must be used with clinical assessment.
\end{abstract}

Key words: Inferior vena cava, volume status, acute kidney injury, ultrasonography

Corresponding author: Kenneth Nugent, MD

Contact Information: Kenneth.nugent@ttuhsc.edu

DOI: 10.12746/swrccc2016.0415.195 


\section{INTRODUCTION}

Acute kidney injury is relatively common in hospitalized patients and clearly increases mortality, the length of stay, and cost. Chertow reported that the adjusted odds ratio for mortality was 6.5 in patients who had an increase in serum creatinine $\geq 0.5 \mathrm{mg} / \mathrm{dL}$ during hospitalization. ${ }^{1}$ The mean adjusted increase in hospital cost was $\$ 7500$ (1998 US dollars). Multiple factors can contribute to the development of acute kidney injury, and clinicians usually focus on volume status, comorbid diseases which the can cause kidney injury, and medications. The assessment of volume status typically involves the use of clinical information, laboratory tests, and possibly venous pressure measurements using central venous catheters. Almost all patients initially receive empiric trials of intravenous fluids. Quantitative measures of volume status could improve this decision making and potentially avoid the adverse consequences of volume overload. The measurement of inferior vena cava (IVC) dimensions and collapsibility has been used in evaluation of patients with trauma, sepsis, and heart failure; these measurements are made easily at the bedside and do not have the risk of invasive procedures. ${ }^{2-4}$ We measured inferior vena cava diameters and collapsibility in patients admitted to our medical intensive care unit with laboratory evidence of presumed acute kidney injury. The goal was to determine IVC dimensions in this group of patients and the association between changes in these dimensions and creatinine following fluid administration.

\section{Methods}

This study prospectively enrolled patients with a presumptive diagnosis of acute kidney injury who were admitted to the medical intensive care unit at University Medical Center in Lubbock, Texas. Patients were screened at 8 AM each day to identify patients with clinical presentations and abnormal laboratory tests consistent with acute kidney injury. A creatinine level greater $\geq 1.5 \mathrm{mg} / \mathrm{dL}$ was considered abnormal enough for inclusion in this study. Current laboratory results were compared with prehospitalization results if available in our electronic medical record. Demographic and clinical information was collected from the medical record on each patient. Inferior vena cava dimensions were obtained at study entry and 24 hours later. In addition, fluid balances and serial creatinine levels were recorded.

Inferior vena cava dimensions were measured using a transverse orientation either at the junction of the hepatic vein with inferior vena cava or $2 \mathrm{~cm}$ below the entry into the right atrium using SonoSite X-PORTE ultrasound equipment (Bothell, WA). The maximum diameter during exhalation and the minimum diameter during inspiration were measured using the M Mode. The patients were supine during these measurements. The percent collapsibility was calculated using 2 formulas. The first formula was: (maximum diameter-minimum diameter)/maximum diameter $X 100$. The second formula was: (maximum diameter- minimum diameter)/ ((maximum diameter+ minimum diameter)/ 2) X 100. These measurements were made by two authors (AY-H and SO) with significant experience with bedside ultrasonography in ICUs.

The investigators were not involved in the management of these patients, and the information collected on inferior vena cava dimensions was not provided to the physicians caring for the patients. All patients gave written and informed consent for participation. This study was approved by the Institutional Review Board at Texas Tech University Health Sciences Center in Lubbock, TX. This study was registered at the clinicaltrials.gov website (NCT 02165072).

Results were summarized using means and standard deviations. Comparisons were made using t-tests or paired t-tests, and correlations were calculated using Spearmen correlation tests. P-values $\leq 0.05$ were considered significant.

\section{RESULTS}

This study enrolled 30 patients with a mean age of $62.4 \pm 16.0$ years; $70 \%$ of the patients were men. The mean body mass index was $30.4 \pm 7.0 \mathrm{~kg} /$ $\mathrm{m} 2$. The patients had the following comorbidities: diabetes-15, hypertension-13, coronary artery disease -5 , atrial fibrillation-5, CHF-3, chronic kidney 
disease-3, acute GI bleed-1, and cirrhosis-0. Eleven patients $(36.7 \%)$ had a prehospitalization creatinine level in our electronic medical records. The mean initial creatinine was $4.3 \pm 4.2 \mathrm{mg} / \mathrm{dL}$; the range was 1.7 $\mathrm{mg} / \mathrm{dL}$ to $22.1 \mathrm{mg} / \mathrm{dL}$. The mean initial fluid balance prior to study entry was $3004 \pm 1590 \mathrm{~mL}$ (Table 1). The mean fractional excretion of sodium was $2.06 \pm 2.65$ $\%$ (25 patients). Six patients had central venous pressure measurements; the lowest reading in individual patients ranged from $1-13 \mathrm{~cm}$ of water, and the highest reading ranged from $8-21 \mathrm{~cm}$ of water. Sixteen patients had echocardiograms during this hospitaliza- tion. The left ventricular ejection fraction was below $40 \%$ in one patient, and the right ventricular systolic pressure was greater than $30 \mathrm{mmHg}$ in six patients. Twenty-one patients had ultrasound of the kidneys at presentation. Seven patients had large kidneys defined by a long axis dimension greater than $12 \mathrm{~cm}$, one patient had hydronephrosis, and three patients had abnormal kidneys based on echogenicity.

Inferior vena cava dimensions are reported in Table 1. The mean maximum dimension was $1.8 \pm$ $0.5 \mathrm{~cm}$ with a range from 0.42 to $2.65 \mathrm{~cm}$. The mean

\begin{tabular}{|l|l|l|}
\hline \multicolumn{2}{|l|}{ Table 1: Creatinine, fluid balances, and IVC dimensions } \\
\hline & Day 0 & Day 1 \\
\hline IVC diameter, expiration, cm & $1.8 \pm 0.5$ & $1.8 \pm 0.4$ \\
\hline IVC diameter, inspiration, cm & $1.2 \pm 0.6$ & $1.3 \pm 0.4$ \\
\hline IVC \% collapse, formula 1* & $32 \pm 20 \%$ & $27 \pm 24 \%$ \\
\hline IVC \% collapse, formula 2** & $41 \pm 28 \%$ & $35 \pm 31 \%$ \\
\hline \multicolumn{2}{|l|}{} \\
\hline Creatinine, mg/dl & $4.3 \pm 4.2$ & $3.64 \pm 4.1^{* * *}$ \\
\hline Fluid balance, ml & $3004 \pm 1590$ & $2500 \pm 2485$ \\
\hline Total fluid balance (2 days), ml/kg & --- & $51 \pm 37$ \\
\hline$*$ Formula 1= (Exp-Insp)/Exp; Formula 2= (Exp-Insp)/((Exp + Insp)/2); $* * * P<0.01$
\end{tabular}

\begin{tabular}{|l|l|}
\hline \multicolumn{2}{|l|}{ Table 2 IVC classification } \\
\hline Guidelines, Amer Soc Echo ${ }^{5}$ & Number \\
\hline$\leq 2.1 \mathrm{~cm},>50 \%$ & 5 \\
\hline$>2.1 \mathrm{~cm},<50 \%$ & 6 \\
\hline Neither & 19 \\
\hline \multicolumn{2}{|l|}{} \\
\hline Brennan et al & Number \\
\hline IVC $>2 \mathrm{~cm}$, Yes & 11 \\
\hline IVC $>2 \mathrm{~cm}$, No & 19 \\
\hline$\%$ collapse $>40 \%$, Yes & 10 \\
\hline$\%$ collapse $>40 \%$, No & 20 \\
\hline & \\
\hline IVC $<2 \mathrm{~cm}, \%$ collapse $>40 \%$ & 6 \\
\hline IVC $>2 \mathrm{~cm}, \%$ collapse $<40 \%$ & 6 \\
\hline Neither & 18 \\
\hline
\end{tabular}


percent collapse was $32 \pm 20 \%$ on day $0 ; 16$ patients (53.3\%) had $\geq 30 \%$ collapse (formula1). The mean percent collapse was $27 \pm 20 \%$ on day $1 ; 13$ patients had a collapse greater than $30 \%$ (formula 1). The distribution of IVC diameters and the percent collapsibility based on 2 separate classification systems are reported in Table 2 . There was no difference in the maximum or minimum diameters using paired t-tests when comparing measurements on day 0 and day 1 . In addition, there was no difference in the percent collapse by either formula when comparing day 0 and day 1 using paired t-tests. There was a significant decrease in serum creatinine levels when comparing day 0 and day 1 results $(p<0.01)$. When the patients were divided into 2 groups based on the median percent change in creatinine, there was no difference in maximum or minimum IVC dimensions or percent collapse between the 2 groups (data not shown). There was a statistically significant correlation between creatinine levels on day 1 and percent collapse (Spearman correlation coefficient: $0.39, \mathrm{P}=0.03$ ) (Figure). When the patients were split into 2 groups based on a fractional excretion of sodium less than or equal to $1 \%$ and a fractional excretion greater than $1 \%$, there were no differences between the minimum IVC diameter and the maximum IVC diameter in these 2 groups (data not shown). There was no difference in the percent collapsibility.
There was a significant negative correlation between the minimum diameter and the percent collapse on day $0(r=-68, P<0.0001)$ and between the minimum diameter and the percent collapse on day 1 $(r=-0.64, P=.0001)$. When the patients were dichotomized into two groups (percent collapse $\leq 30 \%$ and $>$ $30 \%$ ), the minimum diameter on day 0 predicted the percent collapse on day 0 . The cut-off value was 0.94 $\mathrm{cm}$ with an area under the curve of 0.748 . The minimum diameter on day 1 also predicted the percent collapse on day 1 with a cut-off value of $1.24 \mathrm{~cm}$ and an area under the curve of 0.848 .

Two patients died during this hospitalization. Seven patients required dialysis during hospitalization. The pre-discharge creatinine level was below $1.5 \mathrm{mg} / \mathrm{dL}$ in 19 patients.

\section{Discussion}

Most patients in this study had a maximum IVC diameter in the normal range. The average percent collapsibility was slightly greater than $30 \%$. Based on the guidelines for echocardiographic interpretation of the right heart and the Brennan study, most patients fell in the middle group; they were neither definitely hypovolemic nor hypervolemic. ${ }^{5,6}$ The patient

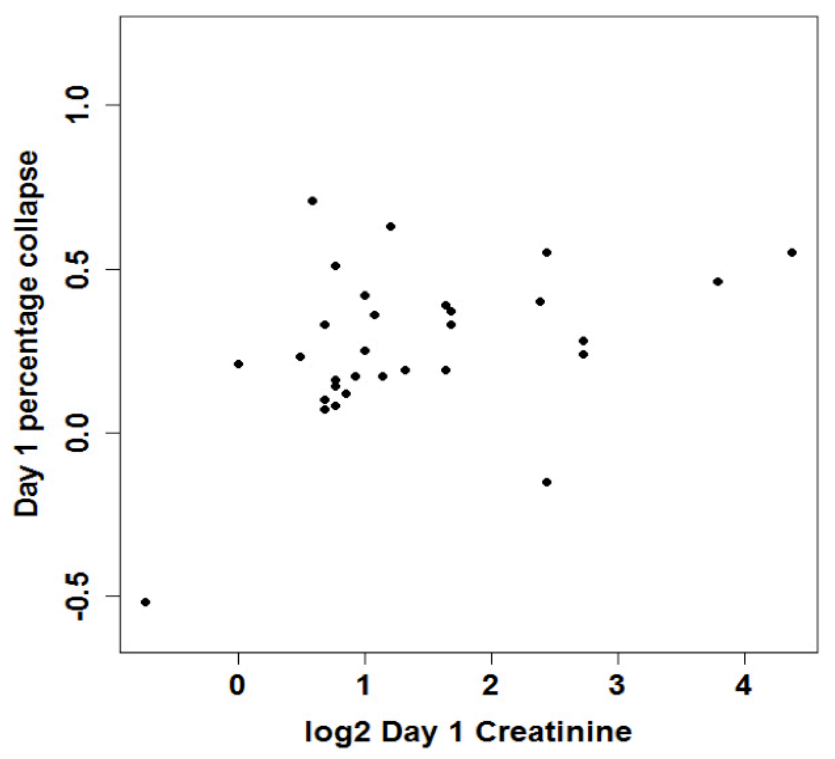


underwent significant volume expansion during first day of this study, but there was little or no change in the mean maximum and minimum diameters on average. In addition, there was no correlation between the change in creatinine levels and IVC dimensions or the fractional excretion of sodium and IVC dimensions.

The measurement of IVC dimensions has been used to evaluate patients in shock secondary to sepsis, patients in critical care units, patients with trauma, and patients with systolic heart failure. There is a reasonably good correlation between IVC dimension measurements and CVP measurements. Barbier and coworkers measured changes in inferior vena cava dimensions, CVP, and cardiac index in 23 patients with sepsis who required mechanical ventilation. $^{2}$ They calculated a distensibility index and determined that there was a good correlation between volume responsiveness defined as an increase in cardiac index $\geq 15 \%$ and the presence of a baseline distensibility index of $18 \%$. These results suggest that measurement of inferior vena cava in critically ill patients can facilitate decisions regarding fluid management. Thanakitcharu measured IVC dimensions in critically ill patients who had the central venous catheters placed. ${ }^{7}$ Patients were classified based on CVP levels as hypovolemic, euvolemic, and hypervolemic. They found a good correlation between the CVP and the collapsibility index of the IVC $(r=-0.612, p<0.001)$. Feissel et al also reported that the respiratory variation in the IVC was a good guide to fluid management in mechanically ventilated patients in shock. ${ }^{8}$ Sefidbakht measured inferior vena cava dimensions in trauma patients who had shock. ${ }^{3}$ Patients in the shock group had significantly smaller diameters than the control trauma patients without shock. The mean collapsibility index was significantly higher in the shock group ( $27 \%$ versus $20 \%, \mathrm{P}<0.001)$. Inferior vena cava measurements are also useful in patients with chronic disease. The mean inferior vena cava dimensions are significantly higher in patients with decompensated congestive heart failure. ${ }^{4}$ In addition, the collapsibility index correlates with the NT-pro BNP level. Guiotto reported that IVC collapsibility could help guide fluid removal by slow continuous ultrafiltration patients with decompensated congestive heart failure. ${ }^{9}$ The IVC collapsibility increased significantly after ultrafiltration and hypotension occurred only in patients who had an IVC collapsibility index greater than $30 \%$. These results suggest that IVC measurements can help determine the volume status in patients with chronic cardiac failure.

There is limited published information on the use of IVC dimensions and collapsibility in hospitalized patients with presumed acute kidney injury. There is more information on IVC measurements in patients undergoing chronic dialysis chronic hemodialysis. Cheriex studied 18 hemodialysis patients and correlated IVC dimensions with right atrial pressure measurements. ${ }^{10}$ Total blood volume in these patients correlated well with IVC dimensions but not with IVC collapsibility. However, there was a poor correlation between dry weight based on clinical assessment and IVC dimensions. Brennan et al used ultrasound measurements to assess intravascular volume status in patients at two hemodialysis clinics. ${ }^{11}$ They found a relatively poor correlation between the patients' weight (at or above dry weight) and IVC dimensions. Thirty-nine percent of the patients were hypovolemic by IVC measurements on arrival to dialysis. In addition, $10 \%$ of the patients who completed dialysis at or below goal weight were still hypervolemic by ultrasound measurements. There was a correlation between hypovolemia measured by IVC and intra-dialysis adverse events, such as hypotension, chest pain, and cramping. The poor correlation between the volume status based on IVC measurements and volume status based on dialysis goals probably reflects the complex comorbidity in these patients, differences in distribution of fluid between vascular spaces, interstitial spaces, and intracellular spaces, and the effect of uremia on vascular function and/or compliance. The fluid volume in vessels is approximately $5 \%$ of the total body fluid volume. Consequently, fluid administration, as in our patients, largely restores the intracellular volume and interstitial volume, and small changes in the vascular volume might not be easily identified with this technology. These measurements likely inform clinical decisions when the patient is definitely either hypovolemic or hypervolemic. The majority of our patients were in between these two states by IVC classification criteria, and management requires clini- 
cal judgment and empiric fluid challenges. In these particular patients end-organ function, including urine output and changes in creatinine, provides important information about the benefits of volume expansion. Sobczyk studied IVC measurements during the first 6 hours in patients who had just completed cardiac surgery. ${ }^{12}$ They found a weak correlation between central venous pressures and IVC derived parameters. In addition, they found no statistically significant differences between patients who were fluid responders and patients were nonresponders in IVC measurements or IVC derived indices. Patients undergoing cardiac surgery often have complex comorbidity and develop a complicated pathophysiologic state during and following surgery on cardiopulmonary bypass. ${ }^{13}$ This clinical situation likely limits the potential correlations between simple anatomic measurements and underlying physiologic state and responses to therapy. Some patients with acute renal failure in our study had chronic comorbidities which influence volume status and vascular responsiveness and acute physiological changes which might limit correlations between IVC measurements and volume status. Information from central venous pressures measurements, renal ultrasound studies, and cardiac echocardiography was not consistently available in our patients. However, the available results did not demonstrate any consistent results or uniform patterns in these patients and did not suggest any management strategies that might differ from clinical assessment.

This study had several limitations. The patient population was heterogeneous with multiple comorbidities and a wide range in creatinine levels. Most patients had an improvement in their creatinine following fluid administration. However, the baseline creatinine measurements were not available in most patients, and long-term follow-up was not available. Some patients likely had chronic kidney disease, and the absolute and percent changes in creatinine based on acute deterioration in kidney function were not ascertainable. However, these patients are the typical patients admitted to ICUs with abnormal kidney function, and clinicians need to make decisions based on information available at presentation.

In summary, we think that simple IVC measurements can facilitate clinical decision making in patients who present with presumed acute kidney injury. We suggest that patients with small IVCs and increased collapse and patients with normal IVCs should benefit from volume expansion. Patients with increased IVC diameters and decreased collapse may be volume overloaded and need reassessment of their clinical status and more cautious fluid challenge trials. Proving these assumptions would require relatively complex clinical trials with objective measurement of volume status and response(s) to fluid administration.

Author Affiliation: Andres Yepes-Hurtado and Khalid Monzer were fellows in Pulmonary and Critical Care Medicine at Texas Tech University Health Sciences Center, Lubbock, TX. Sabry Omar was a resident in Internal Medicine at TTUHSC in Lubbock, TX. Raed Alalawi and Kenneth Nugent are faculty members in Pulmonary and Critical Care Medicine at TTUHSC in Lubbock, TX.

Accepted: 06/10/2016

Reviewers:Vaqar Ahmed MD

Published electronically: 07/15/2016

Conflict of Interest Disclosures: None

\section{REFERENCES}

1. Chertow GM, Burdick E, Honour M, Bonventre JV, Bates DW. Acute kidney injury, mortality, length of stay, and costs in hospitalized patients. Journal of the American Society of Nephrology 2005;16:3365-70.

2. Barbier C, Loubieres Y, Schmit C, et al. Respiratory changes in inferior vena cava diameter are helpful in predicting fluid responsiveness in ventilated septic patients. Intensive Care Medicine 2004;30:1740-6.

3. Sefidbakht S, Assadsangabi R, Abbasi HR, Nabavizadeh A. Sonographic measurement of the inferior vena cava as a predictor of shock in trauma patients. Emergency radiology 2007;14:181-5.

4. Besli F, Kecebas M, Caliskan S, Dereli S, Baran I, Turker Y. The utility of inferior vena cava diameter and the degree of inspiratory collapse in patients with systolic heart failure. American Journal of Emergency Medicine 2015;33:653-7. 5. Rudski LG, Lai WW, Afilalo J, et al. Guidelines for the Echocardiographic Assessment of the Right Heart in Adults: A Report from the American Society of Echocardiography Endorsed by the European Association of Echocardiography, 
a registered branch of the European Society of Cardiology, and the Canadian Society of Echocardiography. Journal of the American Society of Echocardiography 2010;23:685713.

6. Brennan JM, Blair JE, Goonewardena S, et al. Reappraisal of the use of inferior vena cava for estimating right atrial pressure. Journal of the American Society of Echocardiography 2007;20:857-61.

7. Thanakitcharu $P$, Charoenwut M, Siriwiwatanakul N. Inferior vena cava diameter and collapsibility index: a practical non-invasive evaluation of intravascular fluid volume in critically-ill patients. Journal of the Medical Association of Thailand $=$ Chotmaihet thangphaet 2013;96 Suppl 3:S14-22. 8. Feissel M, Michard F, Faller JP, Teboul JL. The respiratory variation in inferior vena cava diameter as a guide to fluid therapy. Intensive Care Medicine 2004;30:1834-7.

9. Guiotto G, Masarone M, Paladino F, et al. Inferior vena cava collapsibility to guide fluid removal in slow continuous ultrafiltration: a pilot study. Intensive Care Medicine 2010;36:692-6.

10. Cheriex EC, Leunissen KM, Janssen JH, Mooy JM, van Hooff JP. Echography of the inferior vena cava is a simple and reliable tool for estimation of 'dry weight' in haemodialysis patients. Nephrology, dialysis, transplantation : official publication of the European Dialysis and Transplant Association - European Renal Association 1989;4:563-8.

11. Brennan JM, Ronan A, Goonewardena S, et al. Handcarried ultrasound measurement of the inferior vena cava for assessment of intravascular volume status in the outpatient hemodialysis clinic. Clinical Journal of the American Society of Nephrology 2006;1:749-53.

12. Sobczyk D, Nycz K, Andruszkiewicz P. Bedside Ultrasonographic Measurement of the Inferior Vena Cava Fails to Predict Fluid Responsiveness in the First 6 Hours After Cardiac Surgery: A Prospective Case Series Observational Study. Journal of Cardiothoracic and Vascular Anesthesia 2015;29:663-9.

13. Omar $S$, Zedan A, Nugent K. Cardiac Vasoplegia Syndrome: Pathophysiology, Risk Factors and Treatment. American Journal of the Medical Sciences 2015;349:80-8. 